\title{
Astragalus polysaccharides affect insulin resistance by regulating the hepatic SIRT1-PGC-1 $\alpha /$ PPAR $\alpha$-FGF21 signaling pathway in male Sprague Dawley rats undergoing catch-up growth
}

\author{
CHENGYING GU ${ }^{1}$, YIPENG ZENG ${ }^{2}$, ZHAOSHENG TANG ${ }^{3}$, CHAOXUN WANG $^{1}$, \\ YANJU HE ${ }^{1}$, XINGE FENG ${ }^{2}$ and LIGANG ZHOU ${ }^{1}$
}

\author{
Departments of ${ }^{1}$ Endocrinology and ${ }^{2}$ Traditional Chinese Medicine, Shanghai Pudong Hospital, Fudan University \\ Pudong Medical Center, Shanghai 201399; ${ }^{3}$ Department of Endocrinology, Shanghai East Hospital, \\ Tongji University School of Medicine, Shanghai 200120, P.R. China
}

Received October 12, 2014; Accepted July 17, 2015

DOI: $10.3892 / \mathrm{mmr} .2015 .4245$

\begin{abstract}
The present study investigated the effects of Astragalus polysaccharides (APS) on insulin resistance by modulation of hepatic sirtuin 1 (SIRT1)-peroxisome proliferator-activated receptor (PPAR) $\gamma$ coactivator (PGC)-1 $\alpha /$ PPAR $\alpha$-fibroblast growth factor (FGF)21, and glucose and lipid metabolism. Thirty male Sprague Dawley rats were divided into three groups: A normal control group, a catch-up growth group and an APS-treated (APS-G) group. The latter two groups underwent food restriction for 4 weeks, prior to being provided with a high fat diet, which was available ad libitum. The APS-G group was orally treated with APS for 8 weeks, whereas the other groups were administered saline. Body weight was measured and an oral glucose tolerance test (OGTT) was conducted after 8 weeks. The plasma glucose and insulin levels obtained from the OGTT were assayed, and hepatic morphology was observed by light and transmission electron microscopy. In addition, the mRNA expression levels of PGC-1 $\alpha / \mathrm{PPAR} \alpha$, and the protein expression levels of SIRT1, FGF21 and nuclear factor- $\kappa \mathrm{B}$ were quantified in the liver and serum. APS treatment suppressed abnormal glycolipid metabolism and insulin resistance following 8 weeks of catch-up growth by improving hepatic SIRT1-PPAR $\alpha$-FGF21 intracellular signaling and reducing chronic inflammation, and by partially attenuating hepatic
\end{abstract}

Correspondence to: Professor Ligang Zhou or Dr Chengying Gu, Department of Endocrinology, Shanghai Pudong Hospital, Fudan University Pudong Medical Center, 2800 Gongwei Road, Shanghai 201399, P.R. China

E-mail: ligang.zhou@gmail.com

E-mail: grace6511@163.com

Key words: astragalus polysaccharide, hepatic insulin resistance, sirtuin 1, peroxisome proliferator-activated receptor $\alpha$, peroxisome proliferator-activated receptor $\gamma$ coactivator $1 \alpha$, fibroblast growth factor 21 steatosis. The suppressive effects of APS on liver acetylation and glycolipid metabolism-associated molecules contributed to the observed suppression of insulin resistance. However, the mechanism underlying the effects of APS on insulin resistance requires further research in order to be elucidated. Rapid and long-term treatment with APS may provide a novel, safe and effective therapeutic strategy for type 2 diabetes.

\section{Introduction}

There are two primary risk factors for type 2 diabetes mellitus (T2DM): Genetic predisposition and environmental factors, in which the latter has a more important role. After a period of growth inhibition, the linear growth rate usually exceeds the normal range; this phenomenon, known as catch-up growth, was first described $>40$ years ago by Prader et al (1). Catch-up growth has become the predominant cause for the accelerated spread of T2DM in developing countries (2), such as China, in which rapid economical growth lead to an increase in the prevalence of T2DM in populations who were subjected to nutritional deficiency at a young age. A previous study demonstrated that low birth weight is an independent risk factor for type 2 diabetes in China (3). Further studies demonstrated that catch-up growth following intra-uterine growth retardation in rats (IUGR rats) resulted in increased liver peroxisome proliferator-activated receptor (PPAR)- $\gamma$ coactivator (PGC)-1 promoter histone 3/lysine 9 acetylation levels (4,5), and increased mRNA and protein expression levels of PGC-1 $\alpha$ and PGC-1, resulting in increased hepatic glucose output (6). In addition to causing the epigenetic histone deacetylation changes in IUGR rats, sirtuin 1 (SIRT1) deacetylation also results in the de-acetylation of PGC-1 $\alpha /$ PPAR $\alpha$ and nuclear factor (NF)- $\mathrm{BB}(7)$, as well as other non-histone molecules, thereby improving liver glucose levels, lipid metabolism and inflammation, and regulating insulin secretion. Epidemiological studies regarding catch-up growth are difficult to perform due to the absence of ideal catch-up growth research platforms and data. The high fat diet weight-matching method following a long period or calorie restriction may better reflect the characteristics of a catch-up growth population $(8,9)$. Therefore, the 
study of the association between PGC-1 $\alpha /$ PPAR $\alpha$ acetylation levels, inflammation, steatosis and hepatic insulin resistance in a liver model may further elucidate the high incidence of T2DM in developing countries. An increase in liver SIRT1 deacetylation levels results in abnormal epigenetic changes, improved liver lipid metabolism and reduced inflammation (7), as well as facilitating early prevention of T2DM. Astragalus exerts antioxidative effects (10) and increases neural activation in two important central glucose-sensing regions of the brain (the paraventricular hypothalamus and the nucleus tractus solitarius) thereby augmenting the counterregulatory response to hypoglycemia (11). The present study aimed to investigate the effects of astragalus on the suppression of hypoglycemia via the liver, as well as the underlying mechanism of these effects. APS is an active component of astragalus, which prevents the development of diabetic cardiomyopathy in diabetic rats via the PPAR $\alpha$-mediated regulatory signaling pathway (12). The association between APS and hepatocyte SIRT1-PGC-1 $\alpha /$ PPAR $\alpha$-mediated regulation of hepatic glucose and lipid metabolism, as well as the therapeutic potential of APS in T2DM merits further research.

\section{Materials and methods}

Ethical approval. All experimental procedures were approved by the Ethics of Animal Experiments Committee of the Tongji University School of Medicine (Shanghai, China; approval no. TJMED-012-006). The present study was conducted according to internationally recognized guidelines on animal welfare, as well as the regulations regarding animal welfare in Shanghai, China, and was conducted in accordance with the guidelines of the Chinese Council on Animal Care.

Animals. A total of 28 six-week-old male Sprague-Dawley rats (Center of Experimental Animals, Tongji University School of Medicine, Shanghai, China), weighing 140-160 g, were housed in wire-bottomed cages in $22 \pm 1^{\circ} \mathrm{C}$ with a $12-\mathrm{h}$ light/dark cycle. The rats were raised on a commercial pellet diet (Center of Experimental Animals) consisting of $22 \%$ protein, $66 \%$ carbohydrates and $12 \%$ fat, and were provided with access to tap water ad libitum. The rats were randomly divided into three groups: A normal diet control group ( $\mathrm{NC} ; \mathrm{n}=10)$, a catch-up growth APS-treated group (APS-G; n=9) and a catch-up growth model group (CUGFR; $\mathrm{n}=9$ ). The rats in the $\mathrm{NC}$ group were raised on an ad libitum pellet diet for 8 weeks, and the rats in the CUGFR group were subjected to a dietary restriction for 4 weeks $(60 \%$ of the diet intake of the NC group) following which they were fed with a high fat diet (42\% calories from fat), which was provided ad libitum. The rats in the APS-G group were orally treated with $700 \mathrm{mg} / \mathrm{kg}$ APS (content of effective components, 69\%), purchased from the Beijing Centre Biology Co. Ltd., (Beijing, China), for 8 weeks. The other groups were administrated with saline irrigation at the same dosage. The body weight of each rat was measured every 7 days.

Animal treatment. The rats were subjected to fasting $15 \mathrm{~h}$ prior to intraperitoneal injection with $20 \%$ urethane anesthetic $(5 \mathrm{ml} / \mathrm{kg}$; Shanghai Hengyuan Biological Technology
Co. Ltd, Shanghai, China). The abdominal cavities of the rats were surgically opened following local disinfection. Blood samples $(8-10 \mathrm{ml})$ were obtained from the abdominal aorta, maintained at room temperature for $10 \mathrm{~min}$ prior to centrifugation in an Eppendorf (EP) tube containing EDTA $(1 \mathrm{mg} / \mathrm{ml})$ and then centrifuged for $20 \mathrm{~min}$ at $2000 \mathrm{xg}$. The supernatant was placed into a sterile EP tube and preserved at $-20^{\circ} \mathrm{C}$. The blood samples were used to determine the serum FGF21 levels using an ELISA kit (BioVendor, Asheville, NC, USA). Liver function, blood lipid levels and glycated hemoglobin (HBA1C) levels were also analyzed. Plasma fatty acid, total cholesterol (TC) and triglyceride levels were determined using a NEFAC kit (Wako Pure Chemical Industries, Ltd., Osaka, Japan), which measures oxidase and peroxidase activity (Trinder reaction). Low-density lipoprotein (LDL) and high-density lipoprotein (HDL) were measured using an immunoassay kit (Daiichi Pure Chemicals Co., Ltd., Tokyo, Japan), alanine aminotransferase (ALT) was determined using an IFCC kit (Roche Diagnostics, Basel, Switzerland). HBA1C levels were determined by immunoturbidimetry (cat. no. D-68305; Roche Diagnostics). The rats were fasted for $15 \mathrm{~h}$ prior to intraperitoneal injection of $20 \%$ urethane anesthetic $(5 \mathrm{ml} / \mathrm{kg})$. The abdominal cavities of the rats were surgically opened by pattern clamps and ophthalmology scissors (Shanghai Kang Chau Medical Instrument Co., Ltd, Shanghai, China) followed by local ethanol disinfection (Sinopharm Co., Ltd., Shanghai, China). Subsequently, blood was drawn from the abdominal aorta and anti-coagulated with EDTA $(1 \mathrm{mg} / \mathrm{ml})$. Plasma was obtained by centrifuging the blood at $2000 \mathrm{xg}$ at $4^{\circ} \mathrm{C}$ for $20 \mathrm{~min}$ and frozen at $20^{\circ} \mathrm{C}$ for further analyses. The liver, epididymal adipose and perirenal fat tissues were separated and weighed under sterile conditions after the rats were sacrificed by decapitation. The liver tissue samples were immediately dissected, fixed in $2 \%$ glutaraldehyde (Sinopharm Co., Ltd) and 10\% formalin or frozen in liquid nitrogen until further analysis. The liver index (the ratio of liver weight to total body weight of the rats) and internal body fat ratio (epididymal fat weight plus perirenal fat weight) were calculated.

Oral glucose tolerance test (OGTT) and lipid parameters. An OGTT test was conducted as follows: A total of $2 \mathrm{~g}$ glucose/100 g body weight was orally administered following overnight fasting for $15 \mathrm{~h}$. Blood samples were collected from the rat tail venous plexus $0,15,30,60$ and $120 \mathrm{~min}$ after glucose treatment, in order to measure the blood glucose and plasma insulin concentration levels. Blood glucose was measured by a glucometer (Roche Accu-Chek Performa; Roche Diagnostics). The blood was then centrifuged and placed into an EP tube containing $1 \mathrm{mg} / \mathrm{ml}$ EDTA (Shanghai Zurui Biological Technology Co., Ltd, Shanghai, China), in order to measure the blood insulin levels using an ELISA kit (EMD Millipore, Billerica, MA, USA). The plasma was then either immediately used for experimentation or frozen at $-20^{\circ} \mathrm{C}$ for further analyses.

Western blot analysis. The frozen rat liver tissue samples were homogenized three times, for $15 \mathrm{sec}$, in ice-cold radioimmunoprecipitation assay homogenization buffer (Merck Chemical Technology Co. Ltd, Shanghai, China) 
containing Protease Inhibitor Cocktail Set III (cat. no. 539134; Invitrogen Life Technologies, Inc., Carlsbad, CA, USA) and Phosphatase Inhibitor Cocktail Set V (cat. no. 524629; Invitrogen Life Technologies, Inc.) using a PRO 200 homogenizer (PRO Scientific, Inc., Oxford, CT, USA). The homogenate was centrifuged at $4^{\circ} \mathrm{C}$ for $30 \mathrm{~min}$ at $12,000 \mathrm{x} \mathrm{g}$ and the supernatant was used as the total protein concentration. The total protein concentration then underwent protein quantification according to the Bradford method (Protein Assay kit II; cat no. 5000002; Bio-Rad Laboratories, Inc., Hercules, CA, USA) according to the manufacturer's instructions. Protein assay dye reagent concentrate (Bio-Rad) and BSA (Thermo Fisher Scientific, Waltham, MA, USA) were used as the protein standard. Protein $(40 \mu \mathrm{g})$ was separated by $4-12 \%$ polyacrylamide gels [depending on the protein of interest; NuPAGE 4-12\% Bis-Tris Gel $1.0 \mathrm{mmx} 12$ well; MOPS SDS running buffer (20X); both from Invitrogen Life Technologies, Inc.] and transferred onto nitrocellulose membranes (iBlot transfer stacks nitrocellulose, regular; Invitrogen Life Technologies) using a semidry transfer protocol. Precision Plus Protein Dual Color standards (Bio-Rad) and Novex ${ }^{\circledR}$ Sharp Pre-stained Protein standard (Invitrogen) were used as the protein ladder. Following membrane blocking with Odyssey Blocking Buffer (LI-COR Biotechnology, Lincoln, NE, USA), the membranes were incubated with the appropriate primary antibody: Rabbit anti-NF- $\kappa$ B p65 polyclonal immunoglobulin (Ig)G (1:200; cat. no. sc-372; Santa Cruz Biotechnology, Inc., Dallas, TX, USA), mouse anti-SIRT1 monoclonal antibody (1:2,000; cat. no. ab110304; Abcam, Cambridge, UK), and rabbit anti-human FGF21 polyclonal antibody $(0.2-1 \mu \mathrm{g} / \mathrm{ml}$; cat. no. LS-B5864; 1:500 dilution; LifeSpan BioSciences, Inc., Seattle, WA, USA) antibodies, diluted with fluorescently-labeled secondary antibody blocking buffer [IRDye ${ }^{\circledR} 800 \mathrm{CW}$ goat anti-mouse IgG (H+L); 1:10,000; cat. no. 925-32210; or IRDye ${ }^{\circledR} 800 \mathrm{CW}$ goat anti-rabbit IgG (H+L); 1:10,000; cat. no. 925-32211; both from LI-COR Biotechnology). The membranes were then washed twice with phosphate-buffered saline (PBS; Decent Biotech, Göttingen, Germany) with 0.1\% Tween 20 (Sigma, Santa Clara, CA, USA) to remove all residual antibodies, and scanned using an Odyssey Infrared Imaging system with IR fluorescence scan Odyssey 2.1 software (LI-COR Biotechnology). Quantitative values were obtained from the densitometric measurements of the western blotting bands using Image Quant software (Tool, 1D gel analysis; GE Healthcare Bio-Sciences, Pittsburgh, PA, USA).

Reverse transcription-quantitative polymerase chain reaction $(R T-q P C R)$. The liver mRNA expression levels of PGC- $1 \alpha$ and PPAR $\alpha$ were assessed by RT-qPCR. The total RNA was extracted using TRIzol reagent (Invitrogen Life Technologies, Inc.; cat. no. 47122). cDNA was reversetranscribed from $2 \mu \mathrm{g}$ DNase-treated total RNA using an iScript cDNA Synthesis kit (Bio-Rad Laboratories, Inc.; cat no. 170-8891) according to the manufacturer's instructions. The following primer sequences, obtained from Invitrogen Life Technologies, were used: Forward, 5'-CGCACAACTCAGCAAGTCCTC-3' and reverse, 5'-CCTTGCTGGCCTCCAAAGTCTC-3' for rat PGC-1 $\alpha$; and forward, 5'-GTGGCTGCTATAATTTGCTGTG-3' and reverse, 5'-GAAGGTGTCATCTGGATGGGT-3' for PPAR $\alpha$. The cDNA (diluted to $1: 20$ ) served as a template for the RT-qPCR; $100 \mu \mathrm{M}$ primer solution was diluted to $5 \mathrm{pmol} / \mu \mathrm{l}$, and qPCR was performed using SYBR Premix ExTaq (Takara Biotechnology Co., Ltd., Dalian, China). The PCR mixture contained 10.5 $\mu \mathrm{l}$ SYBR Premix ExTaq, $0.5 \mu 1$ of each primer, $1 \mu \mathrm{l}$ cDNA and $7.5 \mu 1$ deionized water. PCR was performed with thermocycling conditions of $95^{\circ} \mathrm{C}$ for $30 \mathrm{sec}, 40$ cycles at $95^{\circ} \mathrm{C}$ for $5 \mathrm{sec}$, and $60^{\circ} \mathrm{C}$ for $30 \mathrm{sec}$ in a DNA cycler apparatus (ABI Prism 7900HT; Applied Biosystems, Thermo Fisher Scientific). Genes were quantified using the $\Delta \Delta \mathrm{Ct}$ method with GAPHD as a housekeeping gene. For each set of reactions, the samples were analyzed in triplicate.

Immunohistochemistry and morphometry. Following sacrifice, the livers of the rats were excised, and the liver wet weights were measured. A section of hepatic tissue $(\sim 3 \times 3 \times 10 \mathrm{~mm})$ was removed via a horizontal incision $1 \mathrm{~cm}$ away from the edge of the right lobe. The hepatic tissue samples were fixed in $10 \%$ formalin, dehydrated with a graded series of ethanol, ethanol uranyl acetate and acetone (all from Sinopharm Co., Ltd.), and stained with hematoxylin and eosin (H\&E; $n=3$ rats/group). Observation and image capture were conducted using a CKX31 light microscope (Olympus Corporation, Tokyo, Japan).

Transmission electron microscopy (TEM). Three 1-mm ${ }^{3}$ liver sections were fixed with $2.5 \%$ glutaraldehyde and stored at $4^{\circ} \mathrm{C}$. The samples were subsequently analyzed at the Electron Microscopy Laboratory at the Shanghai University of Traditional Chinese Medicine (Shanghai, China). Following fixing, samples were rinsed with $0.1 \mathrm{M}$ phosphate buffer and treated with $1 \% \mathrm{OsO}_{4}$ solution (Alfa Aesar Chemical Co., Ltd., Shanghai, China) for $3 \mathrm{~h}$, dehydrated with a graded series of ethanol $(30,50,80$ and $90 \%)$ and propylene oxide, and embedded in epoxy resin (Shanghai Resin Factory Co., Ltd, Shanghai, China). Semi-thin (thickness, $500 \mathrm{~nm}$ ) hepatic sections were stained with $1 \%$ toluidine blue (Sinopharm Co., Ltd) for histopathology. Suitable areas of ultrathin (thickness, 80-90 nm) hepatic sections were selected for ultrastructural analysis. These were sectioned using a diamond knife (diamond knife(cat no. 706602; Leica Microsystems, Wetzlar, Germany), mounted on a copper grid (Shanghai Chemical Experiment Audio Supplies Company Ltd, Shanghai, China), and stained with uranyl acetate and lead citrate. The sections were analyzed using a Tecnai-12 Bio T min TEM (Philips, Amsterdam, Netherlands), and observation and image capture were performed at an acceleration voltage of $80 \mathrm{kV}$.

Statistical analysis. Statistical differences between the groups were evaluated using Student' unpaired t-test. The homeostasis model assessment of insulin resistance (HOMA-IR) was used, where: HOMA-IR = [fasting plasma glucose $(\mathrm{mmol} / \mathrm{l}) \mathrm{x}$ fasting plasma insulin $(\mathrm{mU} / \mathrm{l})] / 22.5$. The data were presented as the mean \pm standard deviation. Data analyses were performed using SPSS 18.0 (SPSS, Inc., Chicago, IL, USA). P $<0.05$ was considered to indicate a statistically significant difference. 


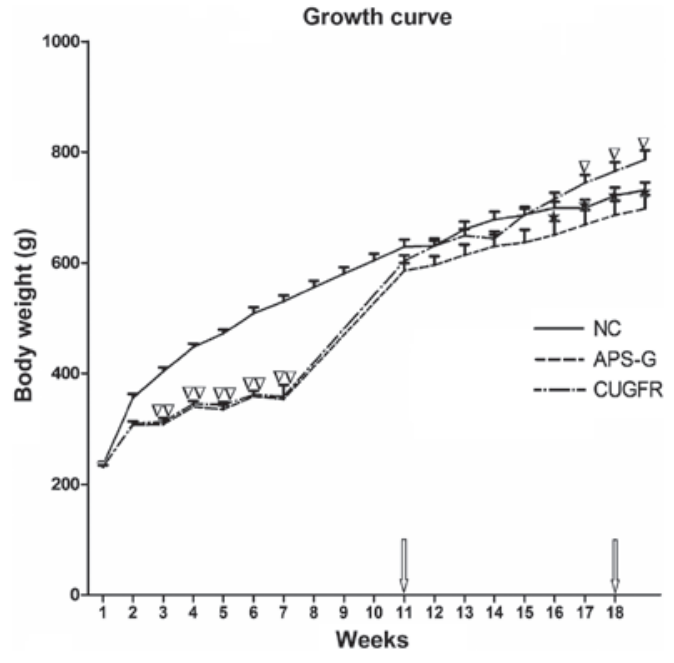

Figure 1. Body weight during food restriction, re-feeding and following treatment. All values are presented as the mean \pm standard deviation $(n=9$ in the APS-G and CUGFR groups; $\mathrm{n}=10$ in the $\mathrm{NC}$ group). ${ }^{\triangleright} \mathrm{P}<0.05$ and ${ }^{\nabla \nabla} \mathrm{P}<0.01$ vs. the NC group; ${ }^{*} \mathrm{P}<0.05$ vs. the CUGFR group. The arrows indicate days when the oral glucose tolerance tests were conducted. NC, normal control group; CUGFR, catch-up growth group; APS-G, Astragalus polysaccharide-treated group.

\section{Results}

Growth of the rats. The growth curve obtained from weighing the rats weekly demonstrated that their growth was arrested during the period of $60 \%$ food restriction, and rapidly increased following re-feeding on a high fat diet for 1 week; however, the rat body weights of the CUGFR group increased less compared with the NC group. The growth curve obtained from the rat weights demonstrated that the APS-G group exhibited a significant trend towards weight loss, as compared with the CUGFR group. Conversely, the rats in the CUGFR group significantly gained weight compared to the rats in the NC group (Fig. 1).

Effects of APS on insulin sensitivity, serum lipid, serum FGF21 and alanine aminotransferase (ALT) levels in high fat diet-fed rats following catch-up growth. Prior to treatment with APS, glucose tolerance curves demonstrated marginally lower (although not statistically significant) serum glucose levels in the CUGFR group rats at 0,15 and 30 min compared with the NC group. At $60 \mathrm{~min}$, following glucose gavage, blood glucose levels were significantly higher than the NC group; these glucose levels failed to return to pre-meal state (Fig. 2A). The insulin curve demonstrated significantly lower insulin levels at $15 \mathrm{~min}$ in the CUGFR group compared with the $\mathrm{NC}$ group $(\mathrm{P}<0.05)$ and that insulin increase was delayed (Fig. 3A). The insulin levels of the APS-G group significantly decreased at $15 \mathrm{~min}$, and the area under the curve of glucose increased, although no statistically significant difference was observed (Fig. 2B, and Fig. 3A and B).

Following treatment with APS, no statistically significant difference was observed between the blood glucose levels of all three groups, and the blood glucose levels of the APS-G rats did not return to their pre-meal state (Fig. $2 \mathrm{C}$ and D). The insulin levels of the CUGFR group significantly increased, when compared with the $\mathrm{NC}$ group $(\mathrm{P}<0.01)$, and treatment with APS moderately reduced this increase, although the results were not statistically significant (Fig. 3D). After $15 \mathrm{~min}$ the levels of insulin were significantly lower in the APS-G group, when compared with the CUGFR group, and no significant decline in these insulin levels occurred throughout the experiment (Fig. 3C). The HOMA-IR and fasting insulin index were significantly higher in the CUGFR group, as compared with the NC group, and following treatment with APS, the two decreased significantly (Table I).

During early catch-up growth, the levels of thyroglobulin (TG), TC, LDL and HBA1C were significantly higher in the CUGFR group as compared with those in the NC group, while levels of HDL were significantly lower than those in the NC group. The levels of free fatty acid (FFA) and ALT in the GUGFR group were not significantly different from those in the NC group. At the late stage of catch-up growth, the levels of LDL, FFA and ALT in the CUGFR group were markedly increased compared with those in the NC group (Table II). No statistically significant increase was observed in the levels of serum lipid and HBA1C following treatment with APS (Table I).

Effects of APS on liver index and visceral fat content ratio of high fat diet-fed rats following catch-up growth. The liver index and visceral fat content ratio of the rats were significantly higher $(\mathrm{P}<0.01$ and $\mathrm{P}<0.05$, respectively) in the CUGFR group, as compared with the NC group. Following 8 weeks of APS treatment, liver weight in the APS-G group reduced significantly compared with the CUGFR group, however the decrease in the liver index was not significant. Although the visceral fat content ratio of the CUGFR group was significantly higher than that of the NC group, treatment with APS did not significantly affect the visceral fat content (Table III).

Effects of APS on hepatic pathology and TEM of high fat diet-fed rats following catch-up growth. Liver H\&E staining demonstrated large quantities of lipid droplet deposition in the liver tissue samples of the CUGFR group, as compared with the NC and APS-G groups. Following treatment with APS, the lipid droplet deposition of the APS-G group decreased, as compared with the CUGFR group, and the tissue samples exhibited lipid droplet fusion (Fig. 4).

TEM examination of the liver samples (Fig. 5) demonstrated that damaged mitochondria and lipid droplets were present in the hepatocytes of the CUGFR (Fig. 5C and D) and APS-G groups (Fig. 5E and F). The groups also exhibited clusters of damaged mitochondria, multi lamellar bodies, and large lipid droplets joined to form large lipid droplets and surrounded by autophagosomal membranes. In the CUGFR group, numerous lipid droplets were present, including fused lipid droplets, and the number of mitochondria and endoplasmic reticula was decreased in the cells. In addition, the cellular structure was severely impaired. Furthermore, the nuclear membranes were not continuous, exhibiting visible holes.

Following treatment with APS, the number of lipid droplets decreased in the APS-G group (Fig. 5E and F), however numerous impaired mitochondria and endoplasmic reticula 

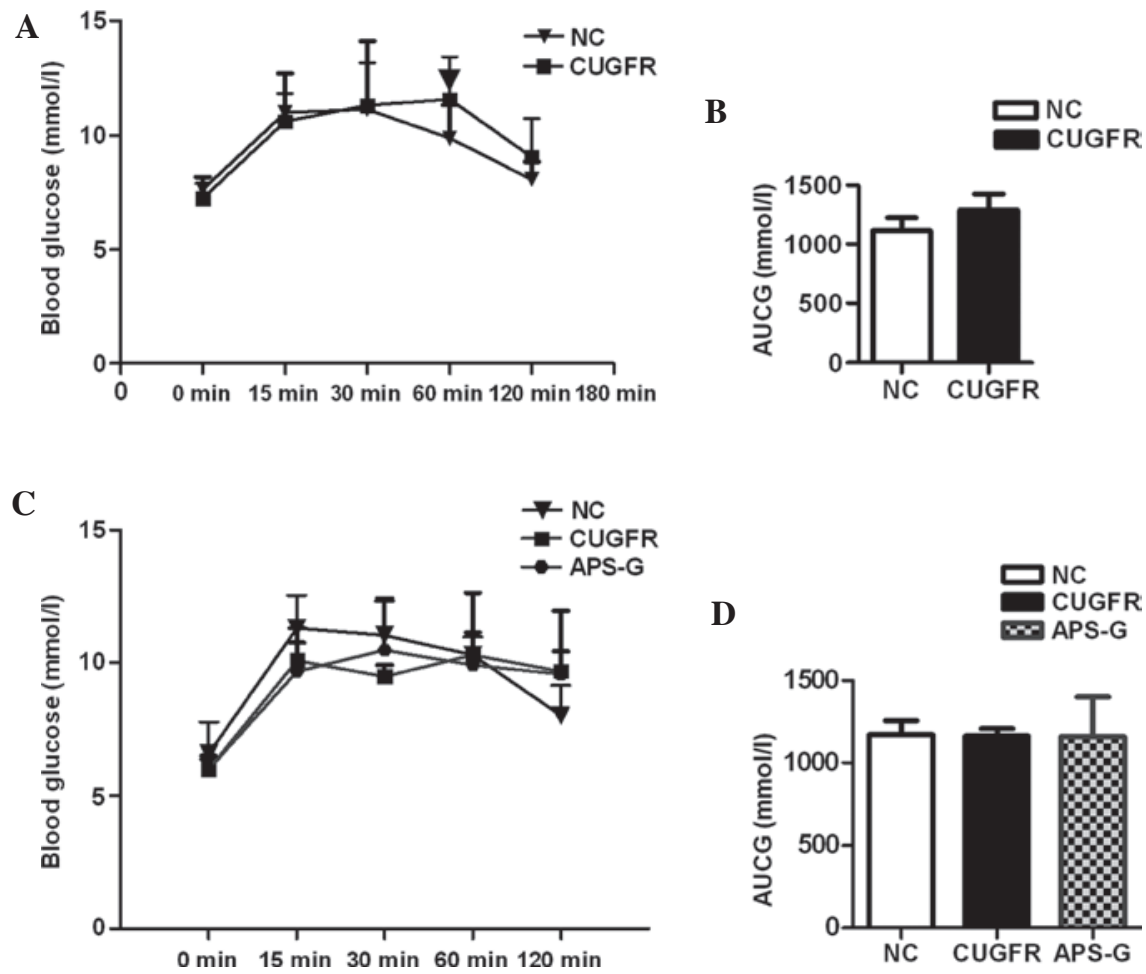

Figure 2. Effects of APS on glucose tolerance and AUCG. Blood glucose levels and AUCG in the NC ( $n=10)$, CUGFR ( $n=9)$ and APS-G ( $n=9)$ groups following a 2-h oral glucose tolerance test, conducted (A and B) 4 weeks following catch-up growth and (C and D) 4 weeks following treatment with APS. The data are presented as the mean \pm standard deviation. 'P $<0.05$ vs. the NC group. APS, Astragalus polysaccharide; NC, normal control group; CUGFR, catch-up growth group; APS-G, APS-treated group; AUCG, area under the curve of glucose.

A

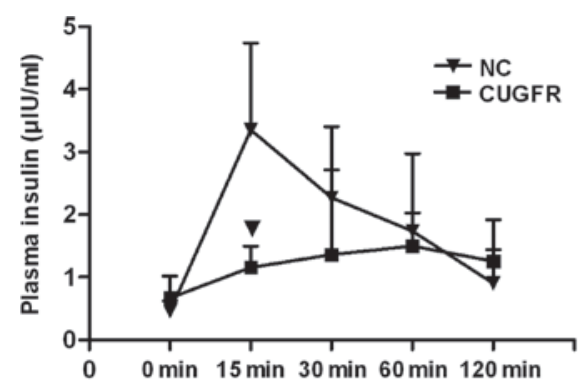

C

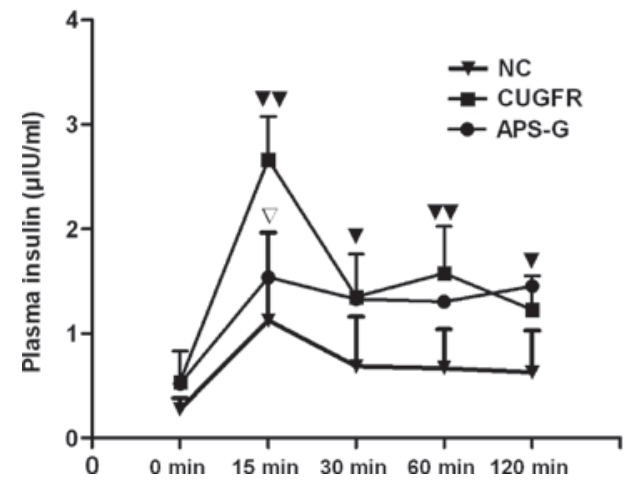

B

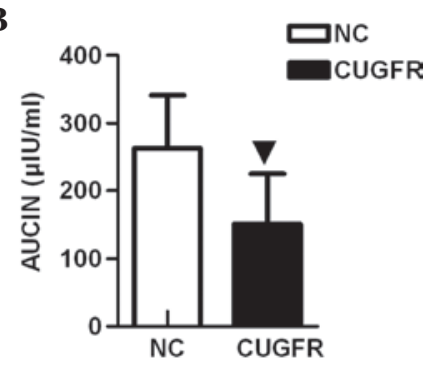

D

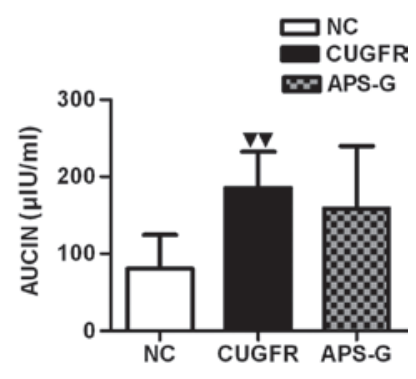

Figure 3. Effects of APS on insulin release and AUCIN. Plasma insulin levels and AUCIN were measured in the NC ( $n=10)$, CUGFR ( $n=9)$ and APS-G ( $n=9)$ groups during a 2-h insulin release test, which was conducted (A and B) 4 weeks after catch-up growth and (C and D) 4 weeks following treatment with APS. The data are presented as the mean \pm standard deviation. ${ }^{\nabla} \mathrm{P}<0.05$ vs. the $\mathrm{NC}$ group; ${ }^{\nabla} \mathbf{\nabla}<0.01$ vs. the $\mathrm{NC}$ group; $\mathbf{\nabla}^{\mathbf{P}}<0.05$ vs. the CUGFR group. APS, Astragalus polysaccharide; NC, normal control group; CUGFR, catch-up growth group; APS-G, APS-treated group; AUCIN, area under the curve of insulin.

remained visible, and the liver cells exhibited expanded endoplasmic reticula and mitochondrial coagulation-associated degeneration. The cell membranes were thick, but continuous (Fig. 5).
Following treatment with APS, the serum FGF21 levels markedly decreased in the CUGFR group, whereas the serum levels of FGF21 in the APS-G group significantly increased compared with the CUGFR group (Fig. 6; $\mathrm{P}<0.05$ ). 
Table I. Serum protein levels and HOMA-IR following treatment with APS.

\begin{tabular}{lcccrr}
\hline Item & NC & CUGFR & APS-G & P1 & P2 \\
\hline TG, mmol/l & $0.76 \pm 0.13$ & $0.81 \pm 0.27$ & $0.70 \pm 0.33$ & 0.504 & 0.521 \\
TC, mmol/l & $1.464 \pm 0.41$ & $1.545 \pm 0.29$ & $1.756 \pm 0.46$ & 0.787 & 0.345 \\
LDL, mmol/l & $0.193 \pm 0.12$ & $0.5 \pm 0.20^{\mathrm{a}}$ & $0.728 \pm 0.28$ & 0.0016 & 0.185 \\
HDL, mmol/l & $1.133 \pm 0.40$ & $0.925 \pm 0.10$ & $0.876 \pm 0.20$ & 0.245 & 0.59 \\
FFA, mmol/l & $0.466 \pm 0.11$ & $0.74 \pm 0.08^{\mathrm{b}}$ & $0.449 \pm 0.14$ & 0.038 & 0.126 \\
ALT, U/l & $37.75 \pm 2.32$ & $58 \pm 26.10^{\mathrm{b}}$ & $63.75 \pm 31.76$ & 0.041 & 0.419 \\
FBG, mmol/l & $6.58 \pm 1.19$ & $6.0 \pm 0.37$ & $6.0 \pm 0.51$ & 0.279 & 1.0 \\
Fins, $\mu$ IU/ml & $5.82 \pm 2.24$ & $12.1 \pm 5.49^{\mathrm{b}}$ & $7.11 \pm 2.09$ & 0.024 & 0.089 \\
HOMA-IR & $1.43 \pm 0.67$ & $3.02 \pm 0.87^{\mathrm{b}}$ & $1.83 \pm 0.42^{\mathrm{c}}$ & 0.026 & 0.049 \\
HBA1C, $\%$ & $3.85 \pm 0.11$ & $3.86 \pm 0.10$ & $3.87 \pm 0.14$ & 0.85 & 0.88 \\
\hline
\end{tabular}

P1, CUGFR vs. NC; P2, APS-G vs. CUGFR. ${ }^{\mathrm{P}} \mathrm{P}<0.01$ and ${ }^{\mathrm{b}} \mathrm{P}<0.05$ vs. NC group; and ${ }^{\mathrm{c}} \mathrm{P}<0.05$ vs. CUGFR. APS, Astragalus polysaccharide; HOMA-IR, homeostasis model assessment of insulin resistance; NC, normal control group; CUGFR, catch-up growth group; APS-G, APS-treated group; HBA1C, glycated hemoglobin; ALT, alanine aminotransferase; TG, thyroglobulin; TC, total cholesterol; LDL, low-density lipoprotein; HDL, high-density lipoprotein; FFA, free fatty acid; FBG, fibrinogen; Fins, fasting insulin.

Table II. HBA1C, serum lipid and ALT levels.

\begin{tabular}{lrrr}
\hline Item & \multicolumn{1}{c}{ NC } & \multicolumn{1}{c}{ CUGFR } & P-value \\
\hline TG, mM/l & $1.0 \pm 0.22$ & $1.49 \pm 0.45^{\mathrm{b}}$ & 0.013 \\
$\mathrm{TC}, \mathrm{mM} / 1$ & $2.17 \pm 0.48$ & $2.77 \pm 0.71^{\mathrm{b}}$ & 0.05 \\
$\mathrm{LDL}, \mathrm{mM} / 1$ & $0.44 \pm 0.21$ & $1.02 \pm 0.43^{\mathrm{a}}$ & 0.002 \\
$\mathrm{HDL}, \mathrm{mM} / 1$ & $0.77 \pm 0.28$ & $0.48 \pm 0.18^{\mathrm{b}}$ & 0.012 \\
FFA, $\mathrm{mM} / \mathrm{l}$ & $1.3 \pm 0.28$ & $1.63 \pm 0.95$ & 0.35 \\
ALT, $\mu \mathrm{M} / 1$ & $47 . \pm 7.13$ & $48.46 \pm 13.6$ & 0.85 \\
HBA1C, $\%$ & $3.99 \pm 0.09$ & $4.18 \pm 0.13^{\mathrm{a}}$ & 0.0039 \\
\hline
\end{tabular}

${ }^{a} \mathrm{P}<0.01$ and ${ }^{\mathrm{b}} \mathrm{P}<0.05$ vs. the NC group. HBA1C, glycated hemoglobin; ALT, alanine aminotransferase; NC, normal control group. CUGFR, catch-up growth group; TG, thyroglobulin; TC, total cholesterol; LDL, low-density lipoprotein; HDL, high-density lipoprotein; FFA, free fatty acid.

Table III. Liver index and visceral fat content ratio of the rats.

\begin{tabular}{|c|c|c|c|c|c|}
\hline Item & $\mathrm{NC}$ & CUGFR & APS-G & $\mathrm{P} 1$ & $\mathrm{P} 2$ \\
\hline Weight, g & $774.5 \pm 39.2$ & $786 \pm 48.3$ & $697.8 \pm 67.7^{c}$ & 0.101 & 0.019 \\
\hline Liver weight, g & $17.14 \pm 1.78$ & $31.67 \pm 2.47^{\mathrm{b}}$ & $27.49 \pm 4.05^{\mathrm{c}}$ & $<0.001$ & 0.046 \\
\hline Liver index & $2.31 \pm 0.25$ & $4.03 \pm 0.17^{\mathrm{b}}$ & $3.94 \pm 0.41$ & $<0.001$ & 0.629 \\
\hline E-P-fat, g & $31.94 \pm 6.41$ & $42.55 \pm 10.62^{\mathrm{a}}$ & $39.36 \pm 13.26$ & 0.038 & 0.638 \\
\hline V-fat, weight $\%$ & $4.52 \pm 0.53$ & $5.75 \pm 0.99^{a}$ & $5.19 \pm 1.35$ & 0.018 & 0.446 \\
\hline
\end{tabular}

P1, CUGFR vs. the NC group; P2, APS-G vs. CUGFR. ${ }^{\mathrm{a}} \mathrm{P}<0.05$ and ${ }^{\mathrm{b}} \mathrm{P}<0.01$ vs. the $\mathrm{NC}$ group, ${ }^{\mathrm{c}} \mathrm{P}<0.05$ vs. CUGFR. NC, normal control group; CUGFR, catch-up growth group; APS, Astragalus polysaccharide; APS-G, APS-treated group; E-P, epididymal plus perirenal; V, visceral.

Relative mRNA expression levels of PGC-1 $\alpha$ and PPAR $\alpha$, and protein expression levels of SIRT1, FGF21 and NF- $\kappa B$ in the liver. The expression levels of PGC-1 $\alpha$ markedly increased in the CUGFR group when compared with the NC group, and were marginally increased by treatment with APS (Fig. 7A). The expression levels of PPAR $\alpha$ in the CUGFR group were significantly lower as compared with the NC group, and these expression levels increased following treatment with APS (Fig. 7B; $\mathrm{P}<0.05$ ). In the CUGFR group, the protein expression levels of FGF21 and SIRT1 were significantly lower than the NC group (Fig. 7C and D P<0.05), whereas the expression levels of NF- $\kappa \mathrm{B}$ were significantly increased. In the APS-G group, the previously low expression levels of FGF21 and SIRT1 were increased (with the difference in SIRT1 being 

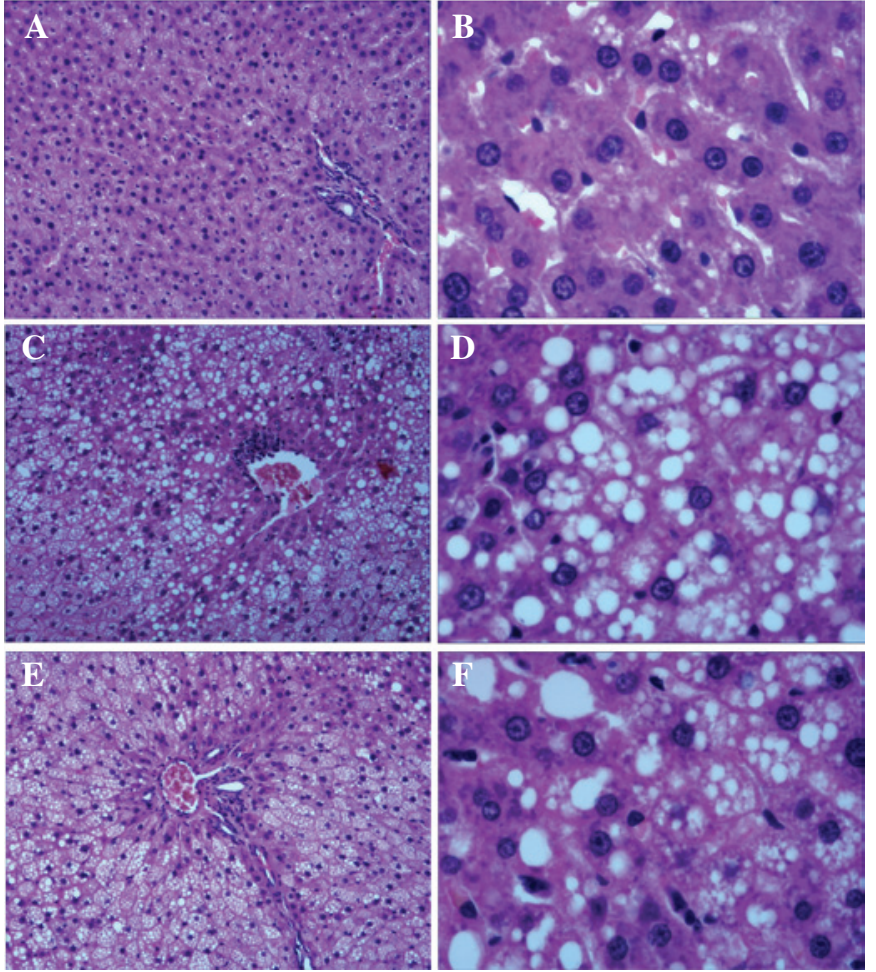

Figure 4. Representative histological observations of hepatic cells in the (A and B) NC, (C and D) CUGFR and (E and F) APS-G groups, as determined by hematoxylin and eosin staining (A, C and E: Magnification, x100; B, D and F: Magnification, $\mathrm{x} 400)$. No histopathological changes were observed in the $\mathrm{NC}$ group, however in the CUGFR group and the APS-G group, cytoplasm vacuolization was observed. The CUGFR group exhibited a large quantity of lipid droplet deposition in the liver tissue samples, as compared with the NC and APS-G groups. The APS-G group exhibited smaller lipid droplets, as compared with the CUGFR group. NC, normal control group; CUGFR, catch-up growth group; APS-G, Astragalus polysaccharide-treated group.

significant compared with the CUGFR group; $\mathrm{P}<0.05$ ). APS treatment appeared to exert no significant effect on the activity

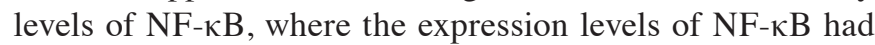
previously been significantly increased in the CUGFR group, as compared with the NC group (Fig. 7E and F).

\section{Discussion}

Catch-up growth may lead to non-alcoholic fatty liver disease (NAFLD), and accumulating evidence suggests that NAFLD is strongly associated with insulin resistance, which is an important factor in the development of T2DM (13). FGF21 regulates hepatic glycolipid metabolism and interacts with PPAR $\alpha(14,15)$, and may serve as a biological target in NAFLD to improve hepatic insulin resistance. SIRT1 is a type of histone deacetylase that regulates $\mathrm{NAD}^{+}(16)$, and regulates certain transcription factors associated with glycolipid metabolism, including PGC-1 $\alpha$ and PPAR $\alpha(17,18)$. Therefore, the present study hypothesized that the SIRT1-PGC-1 $\alpha /$ PPAR $\alpha$-FGF21 signaling pathway may be significant in the pathogenesis of hepatic insulin resistance under catch-up growth. Following diet restriction, the expression levels of SIRT1, PGC-1 $\alpha$ and PPAR $\alpha$ increased significantly, and the levels of hepatic glucose and fatty acid B oxidation increased significantly (19-21). In a previous study, obese mice with genetically-engineered reduced levels of
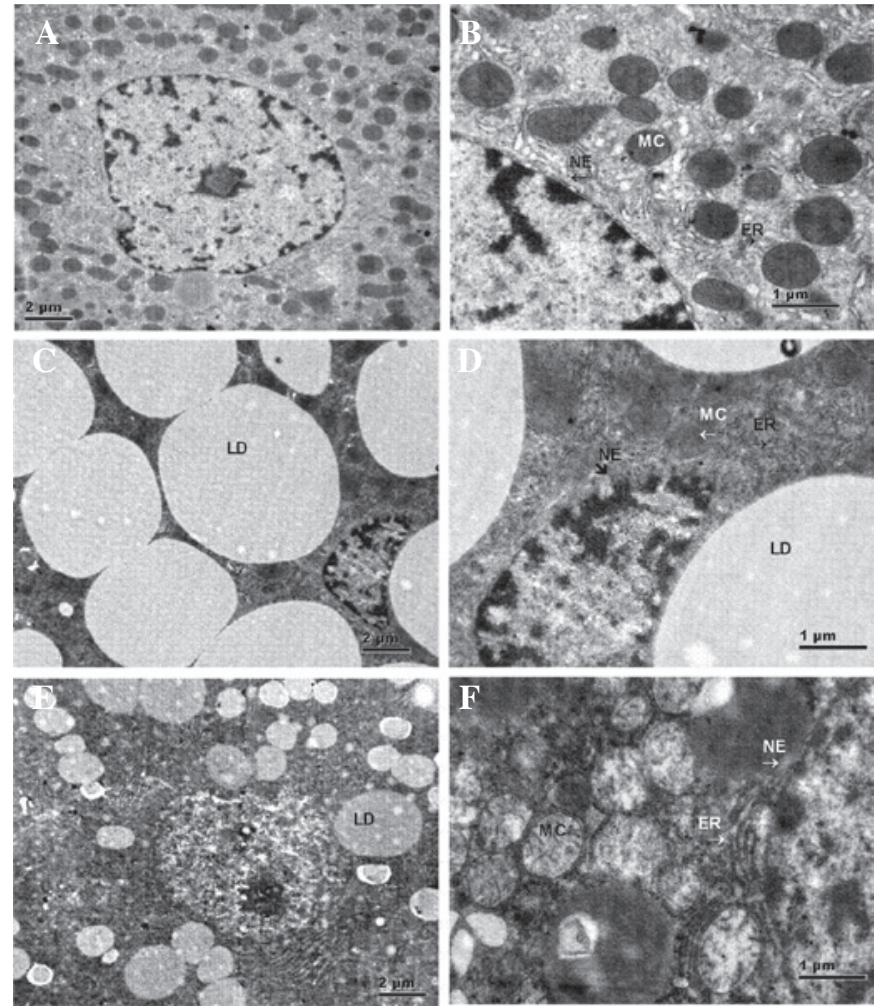

Figure 5. Representative histological observations of hepatic cells in the (A and B) NC, (C and D) CUGFR and (E and F) APS-G groups as determined by transmission electron microscopy (A, C, and E: Magnification, x11,000; scale bar $=2 \mu \mathrm{m}$; B, D, and F: Magnification, $\mathrm{x} 14,000$; scale bar $=1 \mu \mathrm{m}$ ). Hepatic cells of the NC group exhibiting normal MC, ER and NE. Hepatic cells of the CUGFR group exhibiting dilated rough ER, swollen MC (arrow), LD fusion and incomplete NE. Hepatic cells of the APS-G group exhibiting reduced LDs and thick continuous cell membranes. MC, mitochondria; ER, endoplasmic reticulum; NE, nuclear membranes; LD, lipid droplet; NC, normal control group; CUGFR, catch-up growth group; APS-G, Astragalus polysaccharide-treated group.

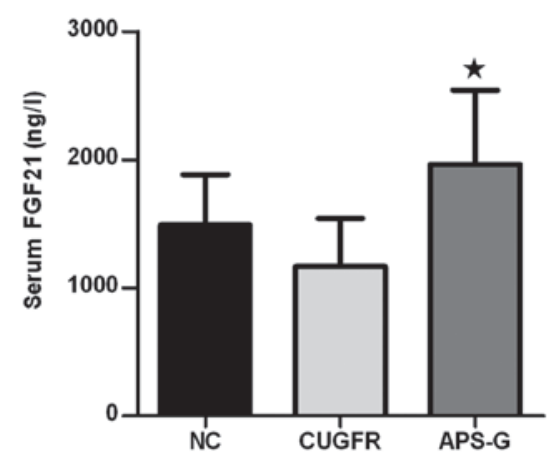

Figure 6. Effects of APS on the serum expression levels of FGF21 in rats subjected to catch-up growth. Circulating levels of FGF21 in $15 \mathrm{~h}$-fasted rats. Error bars represent the mean \pm standard deviation $(n=9$ in the APS-G and CUGFR groups; $\mathrm{n}=10$ in the NC group); ${ }^{\mathrm{P}}<0.05$ vs. CUGFR. APS, Astralagus polysaccharides; FGF21, fibroblast growth factor 21; NC, normal control group; CUGFR, catch-up growth group; APS-G, APS-treated group.

PGC-1 $\alpha$ exhibited improved whole-body insulin sensitivity with increased levels of hepatic and circulating FGF21 (22). In a model of hepatic insulin resistance, glucose absorption was decreased due to the reduction of glucose transporter 1 and the phosphorylation of extracellular signal-regulated kinases $1 / 2$, which were increased by FGF21 $(23,24)$. The 
A

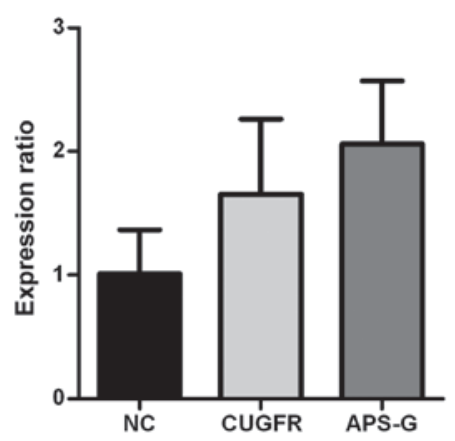

C

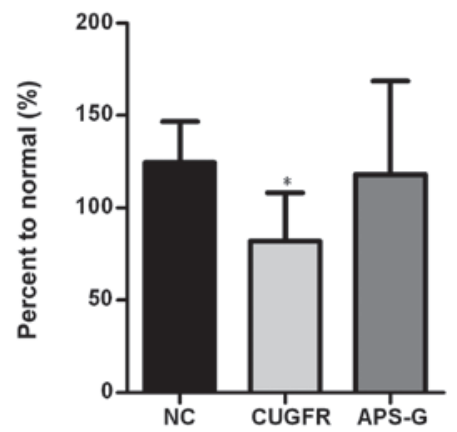

$\mathbf{E}$

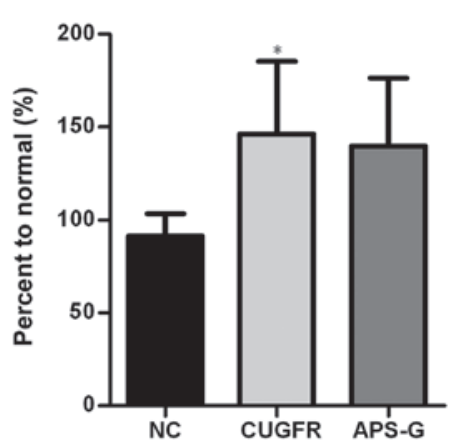

B

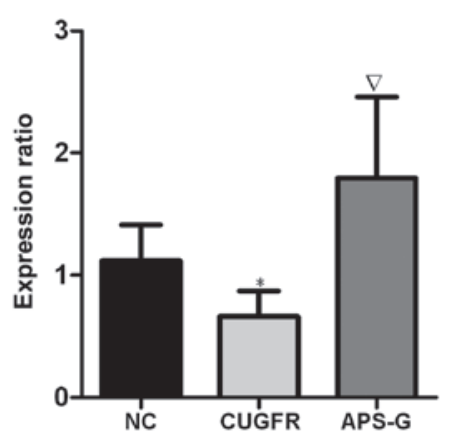

D

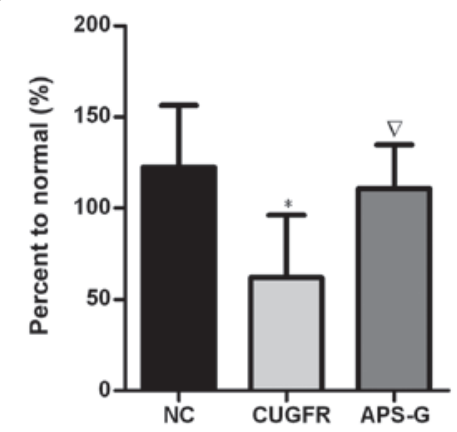

$\mathbf{F}$

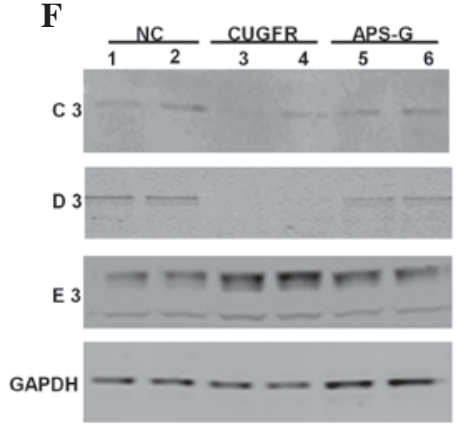

Figure 7. Relative mRNA expression levels of (A) PGC-1 $\alpha$ and (B) PPAR $\alpha$ in the NC group (data are expressed as the mean \pm standard deviation). Catch-up growthinduced alterations in PGC-1 $\alpha$ /PPAR $\alpha$ expression were reversed by treatment with APS. Hepatic protein expression levels of (C) FGF21, (D) SIRT1 and (E) NF- $\mathrm{kB}$ in the NC, CUGFR and APS-G groups fasted for $15 \mathrm{~h}$. (F) Western blot of the NC, CUGFR and APS-G groups fasted for $15 \mathrm{~h}$. C3, D3 and E3 are representative of the western blot lanes of FGF21, SIRT1 and NF-кB, respectively. Lanes 1 and 2, NC group; lanes 3 and 4, CUGFR group; lanes 5 and 6, APS group. High fat diet CUGFR rats, following calorie restriction, exhibited abnormal hepatic protein expression levels of FGF21, SIRT and NF- $\mathrm{B}$, and lower serum expression levels of FGF21, which were improved by treatment with APS. Data are presented as the mean \pm standard error. " $\mathrm{P}<0.05$ vs. the NC group, and ${ }^{\nabla} \mathrm{P}<0.05$ vs. the CUGFR group.

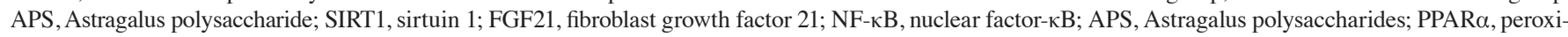
some proliferator-activated receptor $\alpha$; PGC-1 $\alpha$, PPAR $\gamma$ coactivator $1 \alpha$; NC, normal control group; CUGFR, catch-up growth group; APS-G, APS-treated group.

experimental rat model in the present study reflected the status of catch-up growth under a high fat diet following a period of calorie restriction. The results demonstrated that the expression levels of PGC-1 $\alpha$ only marginally increased, whereas the expression levels of PPAR $\alpha$ decreased significantly. Notably, the hepatic protein expression levels of SIRT1 and FGF21 were significantly decreased, with serum expression levels of FGF21 being marginally decreased. Simple variations in the diet, namely a restricted diet followed by a high-fat diet, appeared to result in a catch-up growth effect with significant decreases in SIRT1 and PPARa, while PGC-1 alpha increased slightly. These results demonstrated that the effects on the expression of SIRT1 and its downstream factors (PGC-1 $\alpha / \mathrm{PPAR} \alpha$ ) were more complex than a simple response to high-energy diet following calorie restriction, as the interactions between these factors became influenced by the increased energy levels and onset of catch-up growth. Furthermore, the results of the present study demonstrated that the insulin resistance index, liver index and visceral fat content ratio of the rats significantly increased in the catch-up growth group on the high-energy diet, with liver H\&E staining and TEM indicating the presence of NAFLD pathological changes, which induce hepatic insulin resistance, as well as abnormal glucose and lipid metabolism. In addition, previous studies demonstrated that liver inflammation may cause oxidative stress, resulting in hepatic insulin resistance and NAFLD $(25,26)$. The present study demonstrated that the protein expression levels of $N F-\kappa B$ in the CUGFR rat livers were significantly increased, when compared with the $\mathrm{NC}$ group, which indicated that inflammation contributed to the pathogenesis of NAFLD during the catch-up growth of rats. However, the underlying mechanism of the association 
between SIRT1-PGC-1 $\alpha /$ PPAR $\alpha-F G F 21$ and NF- $\kappa$ B remains unclear and requires further investigation.

Astragalus is a commonly administered type of traditional Chinese medicine and APS is a type of water-soluble polysaccharide that is purified from astragalus. APS exhibits high biological activity $(27)$, regulates inflammation $(28,29)$ and protects the liver $(30,31)$. APS is able to increase insulin sensitivity and lower blood glucose levels by decreasing endoplasmic reticulum stress in liver of patients with T2DM (32). APS treatment reduces the protein expression and activity levels of hepatic glycogen synthase kinase (GSK) $3 \beta$, promotes hepatic insulin signal transduction, and decreases insulin resistance in the liver of diabetic KKAy mice (33). Furthermore, APS promotes the expression of myocardial PPAR $\alpha$, and increases the myocardial lipid metabolism of diabetic hamsters (12). However, to the best of our knowledge, no previous study has observed the effects of APS on the SIRT1-PGC-1 $\alpha /$ PPAR $\alpha-F G F 21$ signaling pathway in the liver of catch-up growth rats.

The results of the present study demonstrate that following treatment with APS, liver cells stained with H\&E and observed by TEM exhibited markedly decreased lipid droplets, and the mitochondria, endoplasmic reticula and cell membrane ultrastructures were improved, as compared with those of the CUGFR group. In addition, the expression level of PPAR $\alpha$ was markedly increased and the change in PGC-1 $\alpha$ expression was not statistically significant. Furthermore, the protein expression levels of SIRT1 and FGF21 were significantly increased. The index of insulin resistance decreased, although not significantly. These results suggest that APS may counteract the pathology of NAFLD, by acting upon liver endoplasmic reticula, and reducing the protein expression and activity levels of hepatic GSK3 $\beta$. Furthermore, APS may prevent NAFLD by increasing insulin sensitivity and promoting hepatic insulin signal transduction $(32,33)$. The results of the present study also suggest that APS may improve hepatic glycolipid metabolism and suppress insulin resistance via modulation of SIRT1-PGC-1 $\alpha /$ PPAR $\alpha-F G F 21$. PGC-1 $\alpha$ enhances the effects of PPAR $\alpha$ through a conformational change, however, the present study demonstrated that APS did not increase the expression levels of PGC-1 $\alpha$ and, therefore, there must be another mechanism that results in the increased protein expression levels of PPAR $\alpha$ and FGF21. Previous studies have reported that SIRT1 increases the expression levels of PPAR $\alpha(34,35)$ and the present study demonstrated that APS increases the protein expression levels of SIRT1. Therefore, the increased expression levels of PPAR $\alpha$ and FGF21 in the liver may be due to an increase in PPAR $\alpha$ deacetylation levels in liver cells; however, this hypothesis requires further investigation. Whether the effects of APS treatment act via the various polysaccharide groups, or via the $\beta$-D- $(1 \rightarrow 3)$ galactan or $\beta$-D- $(1 \rightarrow 6)$ activity groups of galactooligosaccharide side-chains also requires further investigation. In addition, a previous study on the effects of APS on blood glucose in rats demonstrated that APS treatment for 4 weeks exhibited no significant effect on the blood glucose levels of diabetic rats; however, following treatment with APS for 8 weeks, the decreasing effects on blood glucose levels became apparent. Furthermore, no effects were observed in the glucose levels of normal rats (36). Therefore, the results of the present study on the effects of APS on the morphological pathological changes of the liver, and on glucose and lipid metabolism further suggest that the active components of astragalus exerted significant effects on HOMA-IR and fasting insulin levels, although it did not affect hepatic insulin resistance. A study of longer duration would be required to observe the effects of APS on pathological and morphological changes in the liver, as well as on glucose and lipid metabolism. In addition various APS doses and purity levels require investigation to observe their effects on insulin resistance, glycolipid metabolism and NAFLD, to elucidate the ranges and determine guidelines for APS administration to provide early intervention strategies for the prevention of T2DM.

In conclusion, the suppressive effects of APS on liver acetylation levels and on associated glycolipid metabolism molecules contribute to decreasing insulin resistance. However, the mechanism underlying these effects remains to be elucidated, and requires further investigation. Early treatment with APS over longer time periods may provide a novel, safe and effective therapeutic strategy for the treatment of T2DM.

\section{Acknowledgements}

The present study was supported by grants from The National Natural Science Fund (grant no. 81370932) and the Special Fund for the Development of Science and Technology of the Pudong Health Bureau of Shanghai (grant no. PW2012A-29).

The authors of the present study would like to thank Professor Zhixiong Shen of the University of Engineering Science in Shanghai (Shanghai, China) for his contribution to animal experimentation, and to the Pathology Department of Pudong Hospital (Shanghai, China) for hepatic pathological analysis and TEM.

\section{References}

1. Prader A, Tanner JM, von Harnack GA: Catch-up growth following illness or starvation. An example of developmental canalization in man. Pediatrics 62: 646-659, 1963.

2. Chen LL: Catch-up growth - a new territory for understanding insulin resistance. Chin J Endocrinol Metab 24: 235-238, 2008.

3. Xiao XH, Zhang ZX, Cohen HJ, Wang H, Li W, Wang T, Xu T, Liu A, Gai MY, Ying S, et al: Evidence of a relationship between infant birth weight and later diabetes and impaired glucose regulation in a Chinese population. Diabetes Care 31: 483-487, 2008.

4. Fu Q, McKnight RA, Yu X, Wang L, Callaway CW and Lane RH: Uteroplacental insufficiency induces site-specific changes in histone $\mathrm{H} 3$ covalent modifications and affects DNA-histone $\mathrm{H} 3$ positioning in day 0 IUGR rat liver. Physiol Genomics 20: 108-116, 2004.

5. Lane RH, MacLennan NK, Hsu JL, Janke SM and Pham TD: Increased hepatic peroxisome proliferator-activated receptor-gamma coactivator-1 gene expression in a rat model of intrauterine growth retardation and subsequent insulin resistance. Endocrinology 143: 2486-2490, 2002.

6. Yoon JC, Puigserver P, Chen G, Donovan J, Wu Z, Rhee J Adelmant G, Stafford J, Kahn CR, Granner DK, et al: Control of hepatic gluconeogenesis through the transcriptional coactivator PGC-1. Nature 413: 131-138, 2001.

7. Yeung F, Hoberg JE, Ramsey CS, Keller MD, Jones DR, Frye RA and Mayo MW: Modulation of NF-kappa B-dependent transcription and cell survival by the SIRT1 deacetylase. EMBO J 23: 2369-2380, 2004.

8. Nannipieri M, Gonzales C, Baldi S, Posadas R, Williams K, Haffner SM, Stern MP and Ferrannini E; Mexico City diabetes study: Liver enzymes, the metabolic syndrome and incident diabetes: The Mexico City diabetes study. Diabetes Care 28: $1757-1762,2005$ 
9. Cettour-Rose P, Samec S, Russell AP, Summermatter S, Mainieri D, Carrillo-Theander C, Montani JP, Seydoux J, Rohner-Jeanrenaud F and Dulloo AG: Redistribution of glucose from skeletal muscle to adipose tissue during catch-up fat: A link between catch-up growth and later metabolic syndrome. Diabetes 54: 751-756, 2005.

10. Chen R, Shao H, Lin S, Zhang JJ and Xu KQ: Treatment with Astragalus membranaceus produces antioxidative effects and attenuates intestinal mucosa injury induced by intestinalischemia-reperfusion in rats. Am J Chin Med 39: 879-887, 2011.

11. Sang Z, Zhou L, Fan X and McCrimmon RJ: Radix astragali (huangqi) as a treatment for defective hypoglycemia counterregulation in diabetes. Am J Chin Med 38: 1027-1038, 2010.

12. Chen W, Xia Y, Zhao X, Wang H, Chen W, Yu M, Li Y, Ye H and Zhang Y: The critical role of Astragalus polysaccharides for the improvement of PPAR $\alpha$ [correction of PPRA $\alpha$ ]-mediated lipotoxicity in diabetic cardiomyopathy. PLoS One. 7: e45541, 2012.

13. Magee TR, Han G, Cherian B, Khorram O, Ross MG and Desai M: Down-regulation of transcription factor peroxisome proliferator-activated receptor in programmed hepatic lipid dysregulation and inflammation in intrauterine growth-restricted offspring. Am J Obstet Gynecol 199: 271 el-5, 2008.

14. Dushay J, Chui PC, Gopalakrishnan GS, Varela-Rey M, Crawley M, Fisher FM, Badman MK, Martinez-Chantar ML and Maratos-Flier E: Increased fibroblast growth factor 21 in obesity and nonalcoholic fatty liver disease. Gastroenterology 139 : 456-463, 2010

15. Li H, Fang Q, Gao F, Fan J, Zhou J, Wang X, Zhang H, Pan X, Bao Y, Xiang K, et al: Fibroblast growth factor 21 levels are increased in nonalcoholic fatty liver disease patients and are correlated with hepatic triglyceride. J Hepatol 53: 934-940, 2010.

16. Leibiger IB and Berggren PO: Sirt1: A metabilic a master switch that modulates lifespan. Nat Med 12: 34-36, 2006.

17. Gerhart-Hines Z, Rodgers JT, Bare O, Lerin C, Kim SH, Mostoslavsky R, Alt FW, Wu Z and Puigserver P: Metabolic control of muscle mitochondrial function and fatty acid oxidation through SIRT1/PGC-1 alpha. Embo J 26: 1913-1923, 2007.

18. Picard F, Kurtev M, Chung N, Topark-Ngarm A, Senawong T, Machado De Oliveira R, Leid M, McBurney MW and Guarente L: Sirt1 promotes fat mobilization in white adpecytes by repressing PPAR-gamma. Nature 429: 771-776, 2004.

19. Ma L, Dong W, Wang R, Li Y, Xu B, Zhang J, Zhao Z and Wang Y: Effect of caloric restriction on the SIRT1/mTOR signaling pathways in senile mice. Brain Res Bull 116: 67-72, 2015.

20. Zhao Y, Ling F, Griffin TM, He T, Towner R, Ruan H and Sun XH: Up-regulation of the Sirtuin 1 (Sirt1) and peroxisome proliferator-activated receptor $\gamma$ coactivator- $1 \alpha(\mathrm{PGC}-1 \alpha)$ genes in white adipose tissue of Id1 protein-deficient mice: Implications in the protection against diet and age-induced glucose intolerance. J Biol Chem 289: 29112-29122, 2014.

21. Lee J, Hong SW, Park SE, Rhee EJ, Park CY, Oh KW, Park SW and Lee WY: Exendin-4 regulates lipid metabolism and fibroblast growth factor 21 in hepatic steatosis. Metabolism 63: 1041-1048, 2014.
22. Estall JL, Ruas JL, Choi CS, Laznik D, Badman M, Maratos-Flier E, Shulman GI and Spiegelman BM: PGC-1a negatively regulates hepatic FGF21 expression by modulating the heme/Rev-Erbo axis. Proc Natl Acad Sci USA 106: 22510-22515, 2009.

23. Yu D, Sun CY, Sun GP, Ren GP, Ye XL, Zhu SL, Wang WF, Xu PF, Li SJ, Wu Q, et al: The synergistic effect of FGF-21 and insulin on regulating glucose metabolism and its mechanism. Yao Xue Xue Bao 49: 977-984, 2014 (in Chinese).

24. Ge X, Chen C, Hui X, Wang Y, Lam KS and Xu A: Fibroblast growth factor 21 induces glucose transporter-1 expression through activation of the serum response factor/Ets-like protein-1 in adipocytes. J Biol Chem 286: 34533-34541, 2011.

25. Li L, Hai J, Li Z, Zhang Y, Peng H, Li K and Weng X: Resveratrol modulates autophagy and NF- $\mathrm{KB}$ activity in a murine model for treating non-alcoholic fatty liver disease. Food Chem Toxicol 63 166-173, 2013.

26. Gariani K, Philippe J and Jornayvaz FR: Non-alcoholic fatty liver disease and insulin resistance: From bench to bedside. Diabetes Metab 39: 16-26, 2013.

27. Wu F and Chen X: A review of pharmacological study on Astragalus membranaceus (Fisch) Bge. Zhong Yao Cai 27: 232-234, 2004 (In Chinese).

28. Lu J, Chen X, Zhang Y, Xu J, Zhang L, Li Z, Liu W, Ouyang J, Han S and He X: Astragalus polysaccharide induces anti-inflammatory effects dependent on AMPK activity in palmitate-treated RAW264.7 cells. Int J Mol Med 31: 1463-1470, 2013

29. He X, Shu J, Xu L, Lu C and Lu A: Inhibitory effect of Astragalus polysaccharides on lipopolysaccharide-induced TNF-a and IL-1 $\beta$ production in THP-1 cells. Molecules 17: 3155-3164, 2012.

30. Li XT, Zhang YK, Kuang HX, Jin FX, Liu DW, Gao MB, Liu Z and Xin XJ: Mitochondrial protection and anti-aging activity of astragalus polysaccharides and their potential mechanism. Int J Mol Sci 13: 1747-1761, 2012.

31. Wang LX and Han ZW: The effect of astragalus polysaccharide on endotoxin-induced toxicity in mice. Yao Xue Xue Bao 27: 5-9, 1992 (In Chinese)

32. Wang N, Zhang D, Mao X, Zou F, Jin H and Ouyang J: Astragalus polysaccharides decreased the expression of PTP1B through relieving ER stress-induced activation of ATF6 in a rat model of type 2 diabetes. Mol Cell Endocrinol 307: 89-98, 2009.

33. Mao XQ, Wu Y, Wu K, Liu M, Zhang JF, Zou F and Ou-Yang JP: Astragalus polysaccharide reduces hepatic endoplasmic reticulum stress and restores glucose homeostasis in a diabetic KKAy mouse model. Acta Pharmacol Sin 28: 1947-1956, 2007.

34. Chen W, Chen WJ, Xia YP, Lu Y and Yu MH: Effects of astragalus polysaccharides on lipid metabolism and PPAR- $\alpha$ gene expression in myocardium of diabetic hamsters. Fudan Univ J Med Sci 37: 194-197, 2010.

35. Caton PW, Holness MJ, Bishop-Bailey D and Sugden MC: PPAR $\alpha$-LXR as a novel metabolostatic signalling axis in skeletal muscle that acts to optimize substrate selection in response to nutrient status. Biochem J 437: 521-530, 2011.

36. Zou F, Mao XQ, Wang N, Liu J and Ou-Yang JP: Astragalus polysaccharides alleviates glucose toxicity and restores glucose homeostasis in diabetic states via activation of AMPK. Acta Pharmacol Sin 30: 1607-1615, 2009. 